\title{
ON QUASI-GREEDY BASES ASSOCIATED WITH UNITARY REPRESENTATIONS OF COUNTABLE GROUPS
}

\author{
MORTEN NIELSEN ${ }^{\dagger}$ \\ Aalborg University, Denmark
}

\begin{abstract}
We consider the natural generating system for a cyclic subspace of a Hilbert space generated by a dual integrable unitary representation of a countable abelian group. We prove, under mild hypothesis, that whenever the generating system is a quasi-greedy basis it must also be an unconditional Riesz basis. A number of applications to Gabor systems and to general Vilenkin systems are considered. In particular, we show that any Gabor Schauder basis that also forms a quasi-greedy system in $L^{2}$ is in fact a Riesz basis, and therefore satisfies the classical Balian-Low theorem.
\end{abstract}

\section{INTRODUCTION}

Let $G$ be a discrete countable abelian group, and suppose that $g \rightarrow T_{g}$ is a unitary representation of $G$ on a Hilbert space $\mathcal{H}$. For fixed $\psi \in \mathcal{H}$, we are interested in properties of the system $\mathcal{Q}:=\left\{T_{g} \psi: g \in G\right\}$ in the cyclic subspace

$$
\mathcal{S}:=\langle\psi\rangle={\overline{\operatorname{Span}\left\{T_{g} \psi: g \in G\right\}}}^{\mathcal{H}} .
$$

Cyclic subspaces are important in many areas of both pure and applied mathematics. One example is in harmonic analysis where systems of the type $\mathcal{Q}$ form the foundation for generating wavelet and Gabor systems, see $[1,5]$.

For representation systems such as wavelet and Gabor systems, the stability and approximation properties of $\mathcal{Q}$ are important, see e.g. [2, $6,12,13]$. In this paper we study approximation properties of systems $\mathcal{Q}$ given that $\mathcal{Q}$ has

2010 Mathematics Subject Classification. 42C10, 41A45.

Key words and phrases. Quasi-greedy bases, dual integrable representation, Gabor systems, integer translates, Vilenkin system.

${ }^{\dagger}$ The author is supported by the Danish Council for Independent Research. Natural Sciences, Grant 12-124675, "Mathematical and Statistical Analysis of Spatial Data". 
some stability properties. More precisely, we suppose that $\mathcal{Q}$, with some fixed ordering, forms a Schauder basis for $\mathcal{S}$ with $\mathcal{Q}$ having the additional property that decreasing rearrangements of expansions relative to $\mathcal{Q}$ are norm convergent. Put another way, we assume that the approximants obtained from $\mathcal{Q}$ by thresholding an expansion in the system converge. Thresholding is a very natural way to build approximants, and for Riesz bases it is known to be (up to a constant) the optimal way to build $m$-terms approximants.

Systems for which decreasing rearrangements are norm convergent are known as quasi-greedy bases. Every unconditional basis is also a quasi-greedy basis, but it is known that conditional quasi-greedy bases exist not only in Banach spaces but also in infinite dimensional Hilbert spaces, see [10] and [17]. However, our main result shows that under mild hypothesis there are no conditional quasi-greedy bases of the type $\mathcal{Q}$ generated by a unitary representation of a countable group. Put another way, if thresholding approximation is convergent, then $\mathcal{Q}$ is an unconditional Riesz basis for $\mathcal{S}$.

One special case related to this setup has been studied earlier. For a cyclic subspace of $L^{2}\left(\mathbb{R}^{d}\right)$ generated by integer translates of a single function, it was shows in [13] that no conditional quasi-greedy bases of integer translates exists. However, the abstract approach in the present paper will show that the result in [13] is just one instance of a more general theory that applies to many other "popular" systems in harmonic analysis such as Gabor and Vilenkin systems. Our general result relies on the notion of a dual integrable representation introduced in [9].

The structure of the paper is as follows. In Section 2 we review some needed background material on quasi-greedy systems in a Hilbert space and on dual integrable representations of locally compact groups. Section 3 contains our main result, Theorem 3.3. Finally, Section 4 and Section 5 contain examples related to Gabor and Vilenkin systems, respectively.

\section{QuAsi-GREedy Systems In A HilberT SPACE}

The present section reviews some needed background material on quasigreedy systems and on dual integrable representations. The dual integrable representations provide a suitable abstract framework for our study in Section 3 of quasi-greedy systems in cyclic subspaces of the type given by Eq. (1.1).

2.1. Quasi-greedy systems in a Hilbert space. An ordered family $\mathcal{B}=\left\{x_{n}\right.$ : $n \in \mathbb{N}\}$ of vectors in a Hilbert space $\mathcal{H}$ is called a Schauder basis for $\mathcal{H}$ if there exists a unique sequence of continuous linear functionals $\left\{\alpha_{n}\right\}_{n \in \mathbb{N}}$ on $\mathcal{H}$ such that for every $x \in \mathcal{H}$,

$$
\lim _{N \rightarrow \infty} \sum_{n=1}^{N} \alpha_{n}(x) x_{n}=x
$$


in the norm topology of $\mathcal{H}$. By the Riesz representation theorem there exists a unique vector $y_{n}$ such that $\alpha_{n}(x)=\left\langle x, y_{n}\right\rangle$. It follows that

$$
\left\langle x_{m}, y_{n}\right\rangle=\delta_{m, n}, \quad m, n \in \mathbb{N} .
$$

and that there exists a smallest constant $C=C(\mathcal{B}) \geq 1$ such that, for every $n \in \mathbb{N}$,

$$
1 \leq\left\|x_{n}\right\| \cdot\left\|y_{n}\right\| \leq C .
$$

A pair of sequences $\left(\left\{u_{n}\right\}_{n \in \mathbb{N}},\left\{v_{n}\right\}_{n \in \mathbb{N}}\right)$ in $\mathcal{H}$ is a bi-orthogonal system if $\left\langle u_{m}, v_{n}\right\rangle=\delta_{m, n}, m, n \in \mathbb{N}$. We say that $\left\{v_{n}\right\}_{n \in \mathbb{N}}$ is a dual sequence to $\left\{u_{n}\right\}_{n \in \mathbb{N}}$, and vice versa. A dual sequence is not necessarily uniquely defined. In fact, it is unique if and only if the original sequence is complete in $\mathcal{H}$ (i.e., if the span of the original sequence is dense in $\mathcal{H}$ ). A complete sequence $\left\{x_{n}: n \in \mathbb{N}\right\}$ with dual sequence $\left\{y_{n}\right\}$ is a Schauder basis for $\mathcal{H}$ if and only if the partial sum operators $S_{N}(x)=\sum_{n=1}^{N}\left\langle x, y_{n}\right\rangle x_{n}$ are uniformly bounded on $\mathcal{H}$. Eq. (2.1) shows that every Schauder basis $\left\{x_{n}: n \in \mathbb{N}\right\}$ for $\mathcal{H}$ has an associated bi-orthogonal system $\left(\left\{x_{n}\right\},\left\{y_{n}\right\}\right)$ with a uniquely determined dual sequence. Furthermore, the dual sequence $\left\{y_{n}\right\}$ is also a Schauder basis for $\mathcal{H}$.

We now fix a biorthogonal system $\left(x_{n}, x_{n}^{*}\right)_{n \in \mathbb{N}}$ with $\left\{x_{n}\right\}$ complete in $\mathcal{H}$. We assume that the system is quasi-normalized, i.e., $\inf _{n}\left\|x_{n}\right\|_{\mathcal{H}}>0$ and $\sup _{n}\left\|x_{n}^{*}\right\|_{\mathcal{H}}<\infty$, see (2.2). For each $x \in \mathcal{H}$ and $m \in \mathbb{N}$, we define

$$
\mathcal{G}_{m}(x):=\sum_{n \in A} x_{n}^{*}(x) x_{n},
$$

where $A$ is a set of cardinality $m$ satisfying $\left|x_{n}^{*}(x)\right| \geq\left|x_{k}^{*}(x)\right|$ whenever $n \in A$ and $k \notin A$. Whenever $A$ is not uniquely defined, we arbitrarily pick any such set. The definition of $\mathcal{G}_{m}$ leads directly to the definition of a quasi-greedy system, see [10].

Definition 2.1. A quasi-normalized biorthogonal system $\left(x_{n}, x_{n}^{*}\right)_{n \in \mathbb{N}} \subset$ $\mathcal{H} \times \mathcal{H}$, with $\operatorname{span}_{n}\left(x_{n}\right)$ dense in $\mathcal{H}$, is called a quasi-greedy system if there exists a constant $Q$ such that

$$
\left\|\mathcal{G}_{m}(x)\right\|_{\mathcal{H}} \leq Q\|x\|_{\mathcal{H}}, \quad \forall x \in \mathcal{H}
$$

If the system is also a Schauder basis for $\mathcal{H}$, we will use the term quasi-greedy basis.

REMARK 2.2. It was proved by Wojtaszczyk in [17] that a system is quasigreedy if and only if for each $x \in \mathcal{H}$, the sequence $\mathcal{G}_{m}(x)$ converges to $x$ in norm. 
2.2. Dual integrable representations of $L C A$ groups. We now introduce a suitable setting to study the quasi-greedy property in cyclic subspaces of the type given by Eq. (1.1).

We will use the terminology and notation presented in [9]. Suppose that $G$ is a locally compact abelian (LCA) group. We shall use additive notation for $G$. A character of $G$ is a continuous map $\chi: G \rightarrow\{z \in \mathbb{C}:|z|=1\}$ for which

$$
\chi\left(g_{1}+g_{2}\right)=\chi\left(g_{1}\right) \cdot \chi\left(g_{2}\right), \quad g_{1}, g_{2} \in G .
$$

The character group of $G$, denoted by $\widehat{G}$, is the multiplicative group of all characters of $G$.

A representation of a LCA group $G$ on a Hilbert space $\mathcal{H}$ is a strongly continuous map $g \rightarrow T_{g}$ from $G$ into $\mathcal{L}(H, H)$ of bounded linear operators on $\mathcal{H}$ with bounded inverses such that $T_{g} \circ T_{h}=T_{g+h}$ for all $g, h \in G$. The representation is called unitary if all the operators $T_{g}$ are unitary, i.e., $\left\langle T_{g} \phi, T_{g} \psi\right\rangle=\langle\phi, \psi\rangle$ for all $\phi, \psi \in \mathcal{H}$ and $g \in G$.

Fix a Haar measure $d \chi$ on $\widehat{G}$, and let $g \rightarrow T_{g}$ be a unitary representation of $G$ on a Hilbert space $\mathcal{H}$. This representation is called dual integrable if there exists a function

$$
[\cdot, \cdot]: \mathcal{H} \times \mathcal{H} \rightarrow L^{1}(\widehat{G}, d \chi)
$$

such that

$$
\left\langle\phi, T_{g} \psi\right\rangle=\int_{\widehat{G}}[\phi, \psi] \overline{\chi(g)} d \chi .
$$

An abstract way to interpret dual integrability, which was pointed out in [9], is through Stone's theorem, see e.g. [3, Theorem 4.44]. According to Stone's theorem there is a regular measure $P$ on $\widehat{G}$ with values in the set of self-adjoint projections on $\mathcal{H}$ such that

$$
T_{g}=\int_{\widehat{G}} \chi(g) d P(\chi), \quad g \in G .
$$

For $\phi, \psi \in \mathcal{H}$, the function $\mu_{\phi, \psi}(S):=\langle P(S) \phi, P(S) \psi\rangle$ defines a complex valued measure on $\widehat{G}$ such that

$$
\left\langle\phi, T_{g} \psi\right\rangle=\int_{\widehat{G}} \overline{\chi(g)} d \mu_{\phi, \psi}(\chi) .
$$

Comparing this formula to (2.4), we see that dual integrability happens exactly when $d \mu_{\phi, \psi}$ is absolutely continuous wrt. the Haar measure $d \chi$ in which case $[\phi, \psi]$ is the Radon-Nikodym derivative of $d \mu_{\phi, \psi}$.

It follows from [9, Corollary 2.4] that $[\cdot, \cdot]$ is always a sesqui-linear form and a Hermitian symmetric map. In particular, for a.e. $\chi \in \widehat{G}$,

$$
\left[T_{g} \phi, \psi\right](\chi)=\chi(g)[\phi, \psi](\chi)=\left[\phi, T_{-g} \psi\right](\chi) .
$$


For $\psi \in \mathcal{H} \backslash\{0\}$ one defines $\Omega_{\psi}=\{\chi \in \widehat{G}:[\psi, \psi](\chi)>0\}$. Consider the cyclic space $\langle\psi\rangle={\overline{\left\{T_{g} \psi: g \in G\right\}}}^{\mathcal{H}}$. It is proved in [9] that $S_{\psi}$ defined by

$$
S_{\psi}(\phi):=\chi_{\Omega_{\psi}} \frac{[\phi, \psi]}{[\psi, \psi]}
$$

is a one-to-one isometry from $\langle\psi\rangle$ onto the weighted space

$$
L^{2}\left(\Omega_{\psi},[\psi, \psi](\chi) d \chi\right) .
$$

2.3. Countable abelian groups. We now focus on the restricted case where $G$ is a countable abelian group with the discrete topology. We assume that $G$ is ordered according to $G=\left\{g_{n}\right\}_{n=1}^{\infty}$, and we let $g \rightarrow T_{g}$ be a dual integrable representation of $G$ on a Hilbert space $\mathcal{H}$.

For fixed $\psi \in \mathcal{H}$, we consider the system $\mathcal{Q}:=\left\{T_{g_{n}} \psi: n \in \mathbb{N}\right\}$ in the cyclic subspace

$$
\mathcal{S}:=\langle\psi\rangle={\overline{\left\{T_{g_{n}} \psi: n \in \mathbb{N}\right\}}}^{\mathcal{H}} .
$$

We now suppose $\mathcal{Q}$ forms a Schauder basis for $\mathcal{S}$. Then $\mathcal{Q}$ has a uniquely determined bi-orthogonal system and it follows from [9, Proposition 6.1] that $[\psi, \psi](\chi)>0$ for a.e. $\chi \in \widehat{G}$. However, the Schauder basis property is not characterized by positivity of $[\psi, \psi](\chi)>0$, see [14]. We will discuss the special setup where we have either a Gabor system or a shift invariant system in Section 4.

Now we use the isometry $S_{\psi}$ given by (2.5) to translate properties of the system $\mathcal{Q}$ to properties of characters.

Lemma 2.3. Suppose $G=\left\{g_{n}\right\}_{n \in \mathbb{N}}$ is a countable abelian group with $g \rightarrow T_{g}$ a dual integrable representation of $G$ on a Hilbert space $\mathcal{H}$. Let $S_{\psi}$ be defined by (2.5). For any finite scalar sequence $\left\{a_{n}\right\}_{n=1}^{N}$, we have

$$
S_{\psi}\left(\sum_{n=1}^{N} a_{n} T_{g_{n}} \psi\right)(\chi)=\sum_{n=1}^{N} a_{n} \chi\left(g_{n}\right)
$$

Proof. According to (2.4), we have

$$
S_{\psi}\left(T_{g_{k}} \psi\right)(\chi)=\chi\left(g_{k}\right) \frac{[\psi, \psi]}{[\psi, \psi]}=\chi\left(g_{k}\right),
$$

and the result now follows from sesqui-linearity of the bracket $[\cdot, \cdot]$.

Since $S_{\psi}$ is a one-to-one isometry it follows immediately that $\mathcal{Q}$ is a Schauder basis for $\mathcal{S}$ if and only if $\widetilde{\mathcal{Q}}:=\left\{\chi\left(g_{n}\right)\right\}_{n=1}^{\infty}$ is a Schauder basis for $L^{2}(\widehat{G},[\psi, \psi](\chi) d \chi)$. Moreover, $\mathcal{Q}$ is quasi-greedy in $\mathcal{S}$ if and only if $\widetilde{\mathcal{Q}}$ is quasi-greedy in

$$
L^{2}(\widehat{G},[\psi, \psi](\chi) d \chi) .
$$


The Dirichlet kernel associated with $\widetilde{\mathcal{Q}}$ is given by

$$
D_{N}(\chi)=\sum_{n=1}^{N} \chi\left(g_{n}\right),
$$

and it follows that the partial sum operator $\widetilde{S_{N}}$ associated to $\widetilde{\mathcal{Q}}$ is given by

$$
\widetilde{S_{N}}(f)(\alpha)=\int_{\widehat{G}} D_{N}(\alpha-\chi) f(\chi) d \chi
$$

The following property will be essential to prove Theorem 3.3.

Definition 2.4. With the notation considered in Section 2.3, we say that $\widetilde{\mathcal{Q}}$ has the Fejér property provided that the kernels $\left\{F_{N}(\chi):=\left|D_{N}(\chi)\right|^{2} / N\right\}_{N \in \mathbb{N}}$ satisfy the following condition. There exists an increasing sequence $\left\{N_{j}\right\}_{j=1}^{\infty} \subset$ $\mathbb{N}$ such that for every $n \in \mathbb{N}$ and for a.e. $\alpha \in \widehat{G}$,

$$
\lim _{j \rightarrow \infty} \int_{\widehat{G}} F_{N_{j}}(\alpha-\chi) \chi\left(g_{n}\right) d \chi=\alpha\left(g_{n}\right) .
$$

Notice that since $F_{N}$ is non-negative with $\int_{\widehat{G}} F_{N} d \chi=1$, and the characters $\left\{\chi\left(g_{n}\right)\right\}_{n}$ form a complete system in $L^{1}(\widehat{G}, d \chi)$, the Fejér property implies that $\left\{F_{N_{j}}\right\}_{j}$ is an approximate identity for $L^{1}(\widehat{G}, d \chi)$.

\section{Properties of QUASI-GREedy Systems FOR CYCLIC SUBSPACES}

This section contains our main result, Theorem 3.3. The main idea behind Theorem 3.3 is to obtain pointwise information on $\widehat{G}$ for the function $[\psi, \psi]$ by "probing" the function by a suitable approximation of the identity. This idea was introduced in this context in [13]. For another application of this approach see [8, Theorem 4.7]. We first consider some general properties of quasi-greedy system that will be used later to obtain information about the function $[\psi, \psi]$ associated with the cyclic subspace $\mathcal{S}$ given by (1.1). Quasigreedy bases are known to be unconditional relative to sign-changes in the following sense.

Lemma $3.1([17])$. Suppose $\left\{e_{k}\right\}_{k \in \mathbb{N}}$ is a quasi-greedy system in a Hilbert space $\mathcal{H}$. Then there exist constants $0<c_{1} \leq c_{2}<\infty$ such that for every choice of signs $\epsilon_{k}= \pm 1$ and any finite subset $A \subset \mathbb{N}$ we have

$$
c_{1}\left\|\sum_{k \in A} e_{k}\right\|_{\mathcal{H}} \leq\left\|\sum_{k \in A} \epsilon_{k} e_{k}\right\|_{\mathcal{H}} \leq c_{2}\left\|\sum_{k \in A} e_{k}\right\|_{\mathcal{H}}
$$

where $c_{1}$ and $c_{2}$ depend only on the quasi-greedy constant for the system.

For our purpose, Lemma 3.1 is not quite enough. When we consider translates of the Dirichlet kernel, we need to be able to handle arbitrary unimodular complex coefficients and not only \pm 1 as covered by Lemma 3.1. For that purpose, we consider the following proposition, which will be essential for the proof of Theorem 3.3. 
Proposition 3.2. Suppose that $\left\{e_{k}\right\}_{k \in \mathbb{N}}$ is a quasi-greedy system in a Hilbert space $\mathcal{H}$. Then there exist constants $0<c_{1} \leq c_{2}<\infty$ such that for every finite unimodular sequence $\left\{\alpha_{k}\right\}_{k=1}^{N} \subset \mathbb{C}$, we have

$$
c_{1} N^{1 / 2} \leq\left\|\sum_{k=1}^{N} \alpha_{k} e_{k}\right\|_{\mathcal{H}} \leq c_{2} N^{1 / 2} .
$$

Proof. For technical reasons we define a new scalar sequence $\left\{\beta_{k}\right\}_{k \in \mathbb{N}}$ by

$$
\beta_{k}= \begin{cases}\alpha_{k} & 1 \leq k \leq N \\ 1, & k>N\end{cases}
$$

Now observe that $\left\{\beta_{k} e_{k}\right\}_{k \in \mathbb{N}}$ is also a quasi-greedy system in $\mathcal{H}$. By inspection, we see that the greedy approximation operator $\tilde{\mathcal{G}}_{m}$ for $\left.\left\{\beta_{k} e_{k}\right)\right\}_{k \in \mathbb{N}}$ is identical to the approximation operator $\mathcal{G}_{m}$ for $\left\{e_{k}\right\}_{k \in \mathbb{N}}$. This follows from the trivial observation that if $f_{k}$ is the dual element to $e_{k}$, then $\beta_{k} f_{k}$ is the dual element to $\beta_{k} e_{k}$, since $\left|\beta_{k}\right|=1$.

Next we recall that the Hilbert space $\mathcal{H}$ has Rademacher type and cotype 2 , see $[16, \S I I I . A]$. Hence, for any sequence $\left\{f_{\ell}\right\}_{\ell \in \mathbb{N}} \subset \mathcal{H}$, we have the uniform estimate

$$
\int_{0}^{1}\left\|\sum_{\ell=1}^{n} r_{\ell}(t) f_{\ell}\right\|_{\mathcal{H}}^{2} d t \asymp \sum_{\ell=1}^{n}\left\|f_{\ell}\right\|_{\mathcal{H}}^{2},
$$

where $\left\{r_{\ell}\right\}_{\ell \in \mathbb{N}}$ is the sequence of Rademacher functions on $[0,1]$. In particular,

$$
\int_{0}^{1}\left\|\sum_{k=1}^{N} r_{k}(t)\left[\beta_{k} e_{k}\right]\right\|_{\mathcal{H}}^{2} d t=\operatorname{Avg}_{\epsilon_{k}= \pm 1}\left\|\sum_{k=1}^{N} \epsilon_{k}\left[\beta_{k} e_{k}\right]\right\|_{\mathcal{H}}^{2} .
$$

We use (3.4) and (3.3), together with Lemma 3.1 applied to the quasi-greedy system $\left\{\beta_{k} e_{k}\right\}_{k \in \mathbb{N}}$, to obtain

$$
\begin{aligned}
\left\|\sum_{k=1}^{N} \alpha_{k} e_{k}\right\|_{\mathcal{H}}^{2} & \asymp \operatorname{Avg}_{\epsilon_{k}= \pm 1}\left\|\sum_{k=1}^{N} \epsilon_{k}\left[\beta_{k} e_{k}\right]\right\|_{\mathcal{H}}^{2} \\
& \asymp \sum_{k=1}^{N}\left\|\beta_{k} e_{k}\right\|_{\mathcal{H}}^{2} \\
& \asymp N .
\end{aligned}
$$

With Lemma 3.2 in hand we can now turn to our main result.

TheOREM 3.3. Let $G$ be a countable abelian group $G$ with an ordering given by $G=\left\{g_{n}\right\}_{n=1}^{\infty}$. Let $g_{n} \rightarrow T_{g_{n}}$ be a dual integrable unitary representation on a Hilbert space $\mathcal{H}$. Let $\psi \in \mathcal{H}$, and suppose that $\mathcal{Q}=\left\{T_{g_{n}} \psi: n \in \mathbb{N}\right\}$ 
is a quasi-greedy system in the cyclic space $\langle\psi\rangle$. Then $\mathcal{Q}$ is in fact a Riesz basis for $\langle\psi\rangle$, i.e., there exist constants $0<c \leq C<\infty$ such that

$$
c\left\|\left\{c_{n}\right\}\right\|_{\ell_{2}} \leq\left\|\sum_{n \in \mathbb{N}} c_{n} T_{g_{n}} \psi\right\|_{\mathcal{H}} \leq C\left\|\left\{c_{n}\right\}\right\|_{\ell_{2}}
$$

for every finite sequence $\left\{c_{n}\right\}_{n \in \mathbb{N}}$.

Proof. The idea is to consider suitably translated Dirichlet kernels $D_{N}(\chi)=\sum_{n=1}^{N} \chi\left(g_{n}\right), \chi \in \widehat{G}$, since they form "polynomials" with unimodular coefficients. In fact for fixed $\alpha \in \widehat{G}$,

$$
\chi \rightarrow D_{N}(\alpha-\chi)=\sum_{n=1}^{N} \alpha\left(g_{n}\right) \overline{\chi\left(g_{n}\right)},
$$

is a polynomial with unimodular coefficients since $\left|\alpha\left(g_{k}\right)\right|=1$ for every $g_{k} \in$ $G$. Now, according to by Proposition 3.2,

$$
S_{N}:=\sum_{n=1}^{N} \alpha\left(g_{n}\right) T_{g_{n}} \psi
$$

satisfies the norm estimate $\left\|S_{N}\right\|_{\mathcal{H}}^{2} \asymp N$ uniformly in $N$ and $\alpha \in \widehat{G}$. From the linearity and sesqui-linearity of the bracket,

$$
\left[S_{N}, S_{N}\right](\chi)=\left|D_{N}(\alpha-\chi)\right|^{2}[\psi, \psi](\chi) .
$$

The system $\left\{T_{g_{n}} \psi: n \in \mathbb{N}\right\}$ is clearly complete in $\langle\psi\rangle$ since it forms a quasigreedy Schauder basis, and one easily checks that $\Omega_{\psi}=\{\chi \in \widehat{G}:[\psi, \psi](\chi)>$ $0\}=\widehat{G}$. Hence, according to $(2.5)$,

$$
\left\|S_{N}\right\|_{\mathcal{H}}=\left\|S_{\psi}\left(S_{N}\right)\right\|_{L_{2}(\widehat{G},[\psi, \psi] d \chi)},
$$

which implies that,

$$
N \asymp\left\|S_{N}\right\|^{2}=\int_{\widehat{G}}\left|D_{N}(\alpha-\chi)\right|^{2}[\psi, \psi](\chi) d \chi,
$$

uniformly in $N$ and $\alpha \in \widehat{G}$. Then clearly,

$$
1 \asymp \int_{\widehat{G}} \frac{\left|D_{N}(\alpha-\chi)\right|^{2}}{N}[\psi, \psi](\chi) d \chi, \quad N \in \mathbb{N} .
$$

By the Fejér property (2.6), we have that for a.e. $\alpha \in \widehat{G}$,

$$
\int_{\widehat{G}} \frac{\left|D_{N_{j}}(\alpha-\chi)\right|^{2}}{N_{j}}[\psi, \psi](\chi) d \chi \longrightarrow[\psi, \psi](\alpha), \quad \text { as } j \rightarrow \infty,
$$

for some subsequence $\left\{N_{j}\right\}_{j} \subset \mathbb{N}$. It follows at once from (3.7), and the pointwise convergence (3.8), that there exist $c, C>0$ such that

$$
c \leq[\psi, \psi](\alpha) \leq C, \quad \text { a.e. } \alpha \in \widehat{G} \text {. }
$$


It is known that (3.9) implies that $\left\{T_{g_{n}} \psi: n \in \mathbb{N}\right\}$ satisfies (3.6) and the system therefore forms a Riesz basis for $\langle\psi\rangle$, see [9, Proposition 5.2].

\section{EXAMPLES: THE TRIGONOMETRIC SYSTEM}

We first consider two examples related to the trigonometric system in $L^{2}\left(\mathbb{T}^{d}\right)$. The trigonometric system forms the dual group of continuous characters for the group of integers $\mathbb{Z}^{d}$.

The trigonometric system forms an unconditional basis for $L^{2}\left(\mathbb{T}^{d}\right)$, but when a weight is added the situation becomes more complicated. For weighted spaces, the specific ordering of the system may become important. We will focus on the following class of orderings of the trigonometric system.

For $\mathbf{a} \in \mathbb{N}^{d}$ we define the corresponding rectangle by

$$
R_{\mathbf{a}}:=\left\{-a_{1}, \ldots, a_{1}\right\} \times \cdots \times\left\{-a_{d}, \ldots, a_{d}\right\} \subset \mathbb{Z}^{d} .
$$

We say that an ordering $\sigma: \mathbb{N} \rightarrow \mathbb{Z}^{d}$ is adapted to rectangles if there exist an increasing sequence $\left\{N_{j}\right\}_{j=1}^{\infty} \subset \mathbb{N}$, and associated rectangles $R_{\mathbf{a}_{1}} \subset R_{\mathbf{a}_{2}} \subset$ $\cdots$, such that $\mathbb{Z}^{d}=\bigcup_{j} R_{\mathbf{a}_{j}}$ and

$$
\sigma\left(\left\{1,2, \ldots, N_{j}\right\}\right)=R_{\mathbf{a}_{j}}, \quad j=1,2, \ldots
$$

It is not difficult to verify that a trigonometric system $\left\{e^{2 \pi i k \cdot \xi}\right\}_{k \in \mathbb{Z}^{d}}$ with an ordering $\sigma: \mathbb{N} \rightarrow \mathbb{Z}^{d}$ adapted to rectangles satisfies the Fejér condition (2.6). In fact, the associated kernels are separable

$$
F_{N_{j}}(x)=\frac{1}{N_{j}}\left|\sum_{j=1}^{N_{j}} e^{2 \pi i \sigma(j) \cdot x}\right|^{2}=\frac{1}{N_{j}} \prod_{k=1}^{d} \mathcal{F}_{a_{j, k}}\left(x_{k}\right),
$$

with $\mathcal{F}_{n}$ the usual univariate Fejér kernel. Specific examples of orderings $\sigma: \mathbb{N} \rightarrow \mathbb{Z}^{d}$ adapted to rectangles can be found in [11], see also [12].

The Muckenhoupt $A_{2}$ condition for rectangles plays a crucial role when it come to stability of the trigonometric system in weighted $L^{2}$-spaces. We say that a function $w: \mathbb{T}^{d} \rightarrow(0, \infty)$ is an $A_{2, \mathcal{R}}\left(\mathbb{T}^{d}\right)$ weight provided that there exists $C<\infty$ such that for any rectangle $R \subset \mathbb{T}^{d}$,

$$
\frac{1}{|R|} \int_{R} w(x) d x \cdot \frac{1}{|R|} \int_{R} \frac{1}{w(x)} d x \leq C .
$$

4.1. Integer translates. The map $\mathbb{Z}^{d} \ni k \longrightarrow T_{k}$ given by

$$
T_{k} f(x):=f(x+k)
$$


is a unitary representation of the integer group $\left(\mathbb{Z}^{d},+\right)$ on $L^{2}\left(\mathbb{R}^{d}\right)$. The representation is dual integrable, which follows from the observation that

$$
\begin{aligned}
\left\langle\phi, T_{k} \psi\right\rangle_{L^{2}\left(\mathbb{R}^{d}\right)} & =\int_{\mathbb{R}^{d}} \phi(x) \overline{\psi(x+k)} d x \\
& =\int_{\mathbb{R}^{d}} \hat{\phi}(\xi) \overline{\hat{\psi}(\xi)} e^{2 \pi i k \cdot \xi} d \xi \\
& =\int_{[0,1]^{d}}\left\{\sum_{\ell \in \mathbb{Z}^{d}} \hat{\phi}(\xi+\ell) \overline{\hat{\psi}(\xi+\ell)}\right\} e^{2 \pi i k \cdot \xi} d \xi,
\end{aligned}
$$

so one may choose

$$
[\phi, \psi](\xi):=\sum_{\ell \in \mathbb{Z}^{d}} \hat{\phi}(\xi+\ell) \overline{\hat{\psi}(\xi+\ell)}, \quad \phi, \psi \in L^{2}\left(\mathbb{R}^{d}\right) .
$$

Now let $\psi \in L^{2}\left(\mathbb{R}^{d}\right)$. It is known that a sequence of partial sum operators for the trigonometric system $\mathcal{T}:=\left\{e^{2 \pi i k \cdot \xi}\right\}_{k \in \mathbb{Z}^{d}}$ adapted to rectangles is uniformly bounded on $L^{2}\left(\mathbb{T}^{d},[\psi, \psi]\right)$ if $[\psi, \psi]$ satisfies the product $A_{2, \mathcal{R}}$-condition (4.1). With a suitable interpretation a converse statement also holds true, see $[11,12]$ for details. It follows that for a class of orderings of $\mathbb{Z}^{d}$ adapted to rectangles, $[\psi, \psi] \in A_{2, \mathcal{R}}$ is sufficient for the trigonometric system to form a Schauder basis for

$$
L^{2}\left(\mathbb{T}^{d},[\psi, \psi]\right) .
$$

If in addition, $\mathcal{T}$ forms a quasi-greedy basis for $L^{2}\left(\mathbb{T}^{d},[\psi, \psi]\right)$, or equivalently that $\mathcal{S}:=\{\psi(\cdot-k)\}_{k \in \mathbb{Z}^{d}}$ is a quasi-greedy system in $\langle\psi\rangle$, then it follows from Theorem 3.3 that $\mathcal{T}$ (resp. $\mathcal{S}$ ) in fact forms a Riesz basis in $L^{2}\left(\mathbb{T}^{d},[\psi, \psi]\right)$ (resp. in $\langle\psi\rangle$ ). This is exactly the main result obtained in [13].

4.2. Gabor systems. The Gabor representation of the LCA group $\left(\mathbb{Z}^{d} \times\right.$ $\left.\mathbb{Z}^{d},+\right)$ on $L^{2}\left(\mathbb{R}^{d}\right)$ is given by

$$
\mathbb{Z}^{d} \times \mathbb{Z}^{d} \ni(m, n) \longrightarrow T_{m} M_{n} f(x)=f(x+m) e^{2 \pi i n \cdot x},
$$

with $T_{k}$ given by (4.2) and $M_{\ell}$ is the modulation operator $M_{\ell} f(x)=$ $f(x) e^{2 \pi i l \cdot x}$. The Gabor representation is dual integrable through the Zak transform. The Zak transform is an isometry $Z: L^{2}\left(\mathbb{R}^{d}\right) \rightarrow L^{2}\left([0,1]^{d} \times[0,1]^{d}\right)$ given by

$$
Z f(x, \xi)=\sum_{k \in \mathbb{Z}^{d}} f(x+k) e^{2 \pi i k \cdot \xi}, \quad f \in L^{2}\left(\mathbb{R}^{d}\right) .
$$

One easily checks that

$$
Z\left(M_{\ell} T_{k} \psi\right)(x, \xi)=e^{2 \pi i l \cdot x} e^{2 \pi i k \cdot \xi} Z \psi(x, \xi),
$$

and it follows that for $\phi, \psi \in L^{2}\left(\mathbb{R}^{d}\right)$,

$$
\left\langle\phi, M_{\ell} T_{k} \psi\right\rangle_{L^{2}\left(\mathbb{R}^{d}\right)}=\int_{[0,1]^{d}} \int_{[0,1]^{d}} Z \phi(x, \xi) \overline{Z \psi(x, \xi)} e^{-2 \pi i l \cdot x} e^{-2 \pi i k \cdot \xi} d x d \xi .
$$


Consequently, we choose

$$
[\phi, \psi](x, \xi):=Z \phi(x, \xi) \overline{Z \psi(x, \xi)}, \quad \phi, \psi \in L^{2}\left(\mathbb{R}^{d}\right) .
$$

Fix $\psi \in L^{2}\left(\mathbb{R}^{d}\right)$. It was demonstrated for $d=1$ in [7], and for $d>1$ in [11], that $|Z \psi|^{2}(x, \xi) \in A_{2, \mathcal{R}}\left(\mathbb{T}^{d} \times \mathbb{T}^{d}\right)$ is sufficient for the associated Gabor system

$$
\mathcal{G}(\psi):=\left\{M_{\ell} T_{k} \psi\right\}_{\ell, k \in \mathbb{Z}^{d}}
$$

with a suitable ordering adapted to rectangles to form a Schauder basis for $L^{2}\left(\mathbb{R}^{d}\right)$. We can now apply Theorem 3.3 to obtain the following result.

Corollary 4.1. Let $\psi \in L^{2}\left(\mathbb{R}^{d}\right)$. Suppose that the associated Gabor system $\mathcal{G}(\psi)$ forms a quasi-greedy basis for $L^{2}\left(\mathbb{R}^{d}\right)$ with an ordering adapted to rectangles. Then $\mathcal{G}(\psi)$ forms a Riesz basis for $L^{2}\left(\mathbb{R}^{d}\right)$.

An immediate consequence of Corollary 4.1 is that whenever $\mathcal{G}(\psi)$ forms a quasi-greedy basis for $L^{2}\left(\mathbb{R}^{d}\right)$, with an ordering adapted to rectangles, then $\psi \in L^{2}\left(\mathbb{R}^{d}\right)$ must satisfy the Balian-Low theorem, namely

$$
\int_{\mathbb{R}^{d}}|x|^{2}|\psi(x)|^{2} d x \cdot \int_{\mathbb{R}^{d}}|\xi|^{2}|\hat{\psi}(\xi)|^{2} d \xi=+\infty,
$$

due to the fact that $\mathcal{G}(\psi)$ is a Riesz basis.

It is also interesting to note that there exists a conditional Gabor Schauder basis for $L^{2}\left(\mathbb{R}^{d}\right)$ for which the generator does not satisfy the strong form of the Balian-Low theorem (4.3), see [7]. According to Corollary 4.1, such systems cannot form a quasi-greedy basis for $L^{2}\left(\mathbb{R}^{d}\right)$.

\section{EXAMPle: Vilenkin GROUPS}

We now leave the trigonometric system setup and consider an example related to another well known system of group characters, namely the Walsh system.

Let $m=\left(m_{k}\right)_{k \in \mathbb{N}}$ be a sequence of positive integers, and let $\mathbb{Z}_{m_{k}}$ be the $m_{k}$-th discrete cyclic group with Haar measure defined by assigning mass $1 / m_{k}$ to each singleton. The associated Vilenkin group is the product group

$$
\widehat{G_{m}}:=\prod_{k=1}^{\infty} \mathbb{Z}_{m_{k}} .
$$

For each $n \in \mathbb{N}$, and $x=\left(x_{k}\right)_{k \in \mathbb{N}} \in \widehat{G_{m}}$, we set

$$
\rho_{n}(x):=\exp \left(\frac{2 \pi i x_{n}}{m_{n}}\right) .
$$

Clearly, $\rho_{n}$ is a character on $\widehat{G_{m}}$, and it can in fact be shown that any character of $\widehat{G_{m}}$ can be expressed in terms of the $\rho_{n}^{\prime} s$. Put $M_{0}:=1$ and for $k \in \mathbb{N}$ we 
define inductively $M_{k}:=m_{k-1} M_{k-1}$. Then for $n \in \mathbb{N}$ we have a unique finite expansion

$$
n=\sum_{k=0}^{\infty} n_{k} M_{k},
$$

where $0 \leq n_{k}<m_{k}$ for $k=0,1,2, \ldots$. Define $\psi_{0}:=1$, and for $n \in \mathbb{N}$ given by (5.1), we let

$$
\psi_{n}:=\prod_{k=0}^{\infty} \rho_{k}^{n_{k}} .
$$

The system $G_{m}:=\left\{\psi_{k}\right\}_{k=0}^{\infty}$ is called a Vilenkin system and forms a complete orthonormal set of characters for $\widehat{G_{m}}$, see [15]. The well-known Walsh system corresponds to the special case $m_{k}:=2, k \in \mathbb{N}$.

The associated Dirichlet kernel are given by $D_{n}:=\sum_{k=0}^{n-1} \psi_{k}$. The exact pointwise values of $D_{M_{n}}$ are known, see [15, Appendix 0.7], and it can be deduced that $G_{m}$ satisfies the Fejér property for the subsequence with indices $\left\{M_{n}\right\}_{n}$. The question about pointwise convergence a.e. relative to a Vilenkin system (i.e., not only for a subsequence) is more delicate, especially in the so-called unbounded case where $\sup _{k} m_{k}=\infty$, see [4].

The modular representation of $\mathbb{N}_{0}:=\mathbb{N} \cup\{0\}$ on $L^{2}\left(\widehat{G_{m}}, d \chi\right)$ is given by modulation operators

$$
\mathbb{N}_{0} \ni n \rightarrow M_{n} f:=\psi_{n} f, \quad f \in L^{2}\left(\widehat{G_{m}}, d \chi\right) .
$$

The modular representation is dual integrable with $[\phi, \psi]_{M}:=\phi \bar{\psi}$, since the product of any two $L^{2}\left(\widehat{G_{m}}, d \chi\right)$-functions is in $L^{1}\left(\widehat{G_{m}}, d \chi\right)$. Moreover,

$$
\left\langle\phi, M_{n} \psi\right\rangle=\int_{\widehat{G}}[\phi, \psi]_{M} \overline{\psi_{n}(\chi)} d \chi, \quad n \in \mathbb{N}_{0} .
$$

We now apply Theorem 3.3 to obtain the following result, which concludes the paper.

COROLlary 5.1. Let $m=\left(m_{k}\right)_{k \in \mathbb{N}}$ be a sequence of positive integers, and let $\widehat{G_{m}}$ be the associated Vilenkin group. Take $\psi \in L^{2}\left(\widehat{G_{m}}, d \chi\right)$, and suppose that the associated Vilenkin system $G_{m}$ forms a quasi-greedy basis for $\langle\psi\rangle$ generated by the representation (5.3) with the ordering defined by (5.2). Then $G_{m}$ forms a Riesz basis for $\langle\psi\rangle$.

In particular, if $G_{m}$ forms a quasi-greedy basis for $L^{2}\left(\widehat{G_{m}},|\psi|^{2} d \chi\right)$ then $G_{m}$ is a Riesz basis for $L^{2}\left(\widehat{G_{m}},|\psi|^{2} d \chi\right)$ and there exists $c>1$ such that

$$
c^{-1} \leq|\psi(x)| \leq c, \quad \text { a.e. } x \in \widehat{G_{m}} .
$$




\section{REFERENCES}

[1] I. Daubechies, Ten lectures on wavelets, SIAM, Philadelphia, 1992.

[2] C. de Boor, R. A. DeVore and A. Ron, Approximation orders of FSI spaces in $L_{2}\left(\mathbf{R}^{d}\right)$, Constr. Approx. 14 (1998), 631-652.

[3] G. B. Folland, A course in abstract harmonic analysis, CRC Press, Boca Raton, 1995.

[4] G. Gát, Some convergence and divergence results with respect to summation of Fourier series on one and two-dimensional unbounded Vilenkin groups, Ann. Univ. Sci. Budapest. Sect. Comput. 33 (2010), 157-173.

[5] K. Gröchenig, Foundations of time-frequency analysis, Birkhäuser Boston Inc., Boston, 2001.

[6] K. Gröchenig and S. Samarah, Nonlinear approximation with local Fourier bases, Constr. Approx. 16 (2000), 317-331.

[7] C. Heil and A. M. Powell, Gabor Schauder bases and the Balian-Low theorem, J. Math. Phys. 47 (2006), 113506, 21 pp.

[8] E. Hernández, M. Nielsen, H. Šikić and F. Soria, Democratic systems of translates, J. Approx. Theory 171 (2013), 105-127.

[9] E. Hernández, H. Šikić, G. Weiss and E. Wilson, Cyclic subspaces for unitary representations of LCA groups; generalized Zak transform, Colloq. Math. 118 (2010), 313-332.

[10] S. V. Konyagin and V. N. Temlyakov, A remark on greedy approximation in Banach spaces, East J. Approx. 5 (1999), 365-379.

[11] K. Moen, Multiparameter weights with connections to Schauder bases, J. Math. Anal. Appl. 371 (2010), 266-281.

[12] M. Nielsen. On stability of finitely generated shift-invariant systems, J. Fourier Anal. Appl. 16 (2010), 901-920.

[13] M. Nielsen and H. Šikić, Quasi-greedy systems of integer translates, J. Approx. Theory 155 (2008), 43-51.

[14] M. Nielsen and H. Šikić, Schauder bases of integer translates, Appl. Comput. Harmon. Anal. 23 (2007), 259-262.

[15] F. Schipp, W. R. Wade and P. Simon, Walsh series. An introduction to dyadic harmonic analysis, With the collaboration of J. Pál, Adam Hilger Ltd., Bristol, 1990.

[16] P. Wojtaszczyk, Banach spaces for analysts, Cambridge University Press, Cambridge, 1991.

[17] P. Wojtaszczyk, Greedy algorithm for general biorthogonal systems, J. Approx. Theory 107 (2000), 293-314.

M. Nielsen

Department of Mathematical Sciences

Aalborg University

DK-9220 Aalborg

Denmark

E-mail: mnielsen@math.aau.dk

Received: 20.6.2014. 